\title{
REGULAR WALLMAN COMPACTIFICATIONS OF RIM-COMPACT SPACES
}

\author{
OLAV NJÅSTAD
}

(Received 28 July 1980)

Communicated by J. H. Rubinstein

\begin{abstract}
A compact Hausdorf space is regular Wallman if it possesses a separating ring of regular closed sets, an $s$-ring. It was proved by P. C. Baayen and J. van Mill [General Topology and Appl. 9 (1978), 125-129] that if a locally compact Hausdorff space possesses an $s$-ring, then every Hausdorff compactification with zero-dimensional remainder is regular Wallman.

In this paper the reasoning leading to this result is modified to work in a more general setting. Let $\alpha X$ be a Hausdorff compactification of a space $X$, and let $\mathcal{C}_{\alpha}$ be the family of those closed sets in $\alpha X$ whose boundaries are contained in $X$. A main result is the following: If $e_{\gamma} \cap X$ contains an $s$-ring for some Hausdorff compactification $\gamma X$, then every larger Hausdorff compactification $\alpha X$ for which $\mathcal{C}_{\alpha} \cap(\alpha X-X)$ is a base for the closed sets on $\alpha X-X$, is regular Wallman. Various consequences concerning compactifications of a class of rim-compact spaces (called totally rim-compact spaces) are discussed.
\end{abstract}

1980 Mathematics subject classification (Amer. Math. Soc.): 54 D 35.

\section{Introduction}

A compact Hausdorff space is called regular Wallman if it possesses a separating ring of regular closed sets, an $s$-ring. Such a space is a Wallman compactification of every dense subspace. (See [11].) In [1] P. C. Baayen and J. van Mill discuss conditions for a Hausdorff compactification of a completely regular space to be regular Wallman. A main result is the following: If a locally compact space possesses an $s$-ring, then every Hausdorff compactification with zero-dimensional remainder is regular Wallman.

OCopyright Australian Mathematical Society 1981 
In this paper we show how the reasoning leading to this result may be modified to work in a more general setting. A central role is played by the family $e_{\alpha}$ of those closed subsets of the compactification $\alpha X$ whose boundaries are contained in $X$. Among our results is the following: If $\mathcal{C}_{\gamma} \cap X$ contains an $s$-ring for some Hausdorff compactification $\gamma X$, then every larger Hausdorff compactification $\alpha X$ for which $\mathcal{C}_{\alpha} \cap(\alpha X-X)$ is a base for the closed sets of $\alpha X-X$, is regular Wallman. Applications of this result are discussed. In particular the result for locally compact spaces quoted above follows as a rather direct consequence.

\section{Basic concepts}

In the following, $X$ shall always denote a completely regular Hausdorff space. By a compactification we shall always mean a Hausdorff compactification.

Let $\alpha X$ be an arbitrary compactification of $X$. Let $\mathcal{O}_{\alpha}$ and $\mathcal{e}_{\alpha}$ denote the families of open, respectively closed, subsets of $\alpha X$ with boundaries contained in $X$. We note that a set $A$ in $\mathcal{C}_{\alpha}$ is a neighbourhood in $\alpha X$ of every point in $A \cap \rho_{\alpha} X$, where $\rho_{\alpha} X$ denotes the remainder $\alpha X-X$. We shall say that $X$ is rim-included in $\alpha X$ if $\mathcal{\theta}_{\alpha} \cap X$ is a base for the open sets in $X$. (When $X \subset Y$ and $\mathscr{F}$ is a family of subsets of $Y$, we write $\mathscr{F} \cap X$ for $\{F \cap X: F \in \mathscr{F}\}$.) We shall say that $\rho_{\alpha} X$ is rim-excluded in $\alpha X$ if $\mathcal{O}_{\alpha} \cap \rho_{\alpha} X$ is a base for the open sets in $\rho_{\alpha} X$. Clearly if $\rho_{\alpha} X$ is rim-excluded in $\alpha X$, then $\rho_{\alpha} X$ is zero-dimensional (in the sense of small inductive dimension).

We shall write int, $\mathrm{cl}, \partial$ (respectively int ${ }_{\alpha}, \mathrm{cl}_{\alpha}, \partial_{\alpha}$ ) for interior, closure and boundary in $X$ (respectively in $\alpha X$ ).

LEMMA 1. If $\rho_{\alpha} X$ is rim-excluded in $\alpha X$, then $\theta_{\alpha}$ contains a neighbourhood base in $\alpha X$ for every point in $\rho_{\alpha} X$.

Proof. Let $x_{0} \in \rho_{\alpha} X, x_{0} \in A, A$ open in $\alpha X$, and let $W_{1}$ be an open set in $\alpha X$ such that $\alpha X-A \subset W_{1}$, and $x_{0} \notin \mathrm{cl}_{\alpha} W_{1}$. By assumption there is a set $O$ in $\theta_{\alpha}$ such that $x_{0} \in O, \operatorname{cl}_{\alpha} W_{1} \cap \rho_{\alpha} X \subset(\alpha X-O) \cap \rho_{\alpha} X=\left(\alpha X-\operatorname{cl}_{\alpha} O\right) \cap \rho_{\alpha} X$. Clearly the set $W_{2}=\alpha X-\mathrm{cl}_{\alpha} O$ belongs to $\theta_{\alpha}$. Write $U=W_{1} \cup W_{2}$. As $\rho_{\alpha} X \cap \operatorname{cl}_{\alpha} W_{2} \cap O=\varnothing$ by definition, and $\rho_{\alpha} X \cap \operatorname{cl}_{\alpha} W_{1} \cap O \subset \rho_{\alpha} X \cap W_{2} \cap$ $O$, we conclude that $\operatorname{cl}_{\alpha} U \cap \rho_{\alpha} X \cap O=\left(\mathrm{cl}_{\alpha} W_{1} \cup \mathrm{cl}_{\alpha} W_{2}\right) \cap \rho_{\alpha} X \cap O=\varnothing$. Clearly $\partial_{\alpha} U \cap \rho_{\alpha} X \cap(\alpha X-O)=\varnothing$, and consequently $\partial_{\alpha} U \subset X$. It follows that the set $V=\alpha X-\operatorname{cl}_{\alpha} U$ belongs to $\vartheta_{\alpha}$. Now $x_{0} \in V \subset \alpha X-W_{1} \subset A$, from which we conclude that $\theta_{\alpha}$ contains a neighbourhood base for $x_{0}$. This completes the proof. 
LEMMA 2. If $X$ is rim-included in $\alpha X$, then $\Theta_{\alpha}$ contains a neighbourhood base in $\alpha X$ for every point in $X$.

Proof. Let $x_{0} \in X, x_{0} \in A, A$ open in $\alpha X$, and let $B$ be a closed set in $\alpha X$ such that $x_{0} \notin B, \alpha X-A \subset B, \mathrm{cl}_{\alpha}$ int $_{\alpha} B=B$. Let $y \in B$, and let $V$ be an arbitrary neighbourhood of $y$. Then $V \cap$ int $_{\alpha} B \neq \varnothing$, hence $(B \cap X) \cap V \neq \varnothing$, from which follows that $y \in \operatorname{cl}_{\alpha}(B \cap X)$. Thus $B=\operatorname{cl}_{\alpha}(B \cap X)$.

The set $U=(\alpha X-B) \cap X$ is a neighbourhood of $x_{0}$ in $X$, and $U \subset A$. By assumption there is a $W$ in $\theta_{\alpha}$ such that $x_{0} \in W, W \cap X \subset U$. Clearly $W \cap(B \cap X)=\varnothing$, hence also $W \cap \operatorname{cl}_{\alpha}(B \cap X)=\varnothing$. Thus $W \subset \alpha X-$ $\operatorname{cl}_{\alpha}(B \cap X)=\alpha X-B \subset A$, which means that $\theta_{\alpha}$ contains a neighbourhood base for $x_{0}$. This completes the proof.

We recall that $\rho_{\alpha} X$ is said to be zero-dimensionally embedded in $\alpha X$ if $\theta_{\alpha}$ (resp. $e_{\alpha}$ ) is a base for the open (resp. closed) sets in $\alpha X$. A countable remainder is always zero-dimensionally embedded (see [10, p. 273]).

Corollary 1. $\rho_{\alpha} X$ is zero-dimensionally embeded in $\alpha X$ if and only if $X$ is rim-included in $\alpha X$ and $\rho_{\alpha} X$ is rim-excluded in $\alpha X$.

Proof. Follows immediately from Lemma 1 and Lemma 2.

REMARK 1. A locally compact space $X$ is rim-included in every compactification $\alpha X$. The remainder $\rho_{\alpha} X$ (of a locally compact space $X$ ) is rim-excluded in $\alpha X$ if and only if it is zero-dimensional (in which case it is also zero-dimensionally embedded).

We recall that a space is called rim-compact if the family of open (resp. closed) subsets with compact boundaries form a base for the open (resp. closed) sets on $X$. We formulate as a proposition the following fact.

Proposition 1. The space $X$ is rim-compact if and only if it is rim-included in some compactification.

ProOF. The sufficiency follows immediately from Lemma 2 , since boundaries in $\alpha X$ are compact. The necessity follows from the well-known result that a rim-compact space $X$ has at least one compactification in which the remainder is zero-dimensionally embedded, namely the Freudenthal compactification $\phi X$ (see, for example, [4, p. 189], [7, p. 223]). 
We shall briefly illustrate by some examples the concepts we have been discussing so far.

First consider the situation where $X$ is rim-included and $\rho_{\alpha} X$ is rim-excluded in $\alpha X$. Examples of this situation are of course exactly those where the remainder is zero-dimensionally embedded (Corollary 1 ), among those the locally compact spaces with zero-dimensional remainder. In the following example $X$ is not locally compact (though of course rim-compact).

ExAMPLE 1. Let $S$ denote the closed unit square $[0,1] \times[0,1]$ with standard metric topology, let $S^{0}$ denote the open unit square $(0,1) \times(0,1)$, and let $T$ denote the subspace of $S$ consisting of all points both coordinates of which are rational. Define $X=S^{0}-T$, and let $Y$ be the one-point compactification of $S^{0}$. Then $Y$ is a compactification $\alpha X$ of $X$, and $\rho_{\alpha} X$ is easily seen to be zero-dimensionally embedded in $\alpha X$. (Actually $\alpha X$ is the Freudenthal compactification of $X$, see [6, p. 658].)

Also consider the space $X=S-T$ with $\alpha X=S$. Also in this situation $\rho_{\alpha} X$ is zero-dimensionally embedded in $\alpha X$.

Next consider the situation where $X$ is rim-included while $\rho_{\alpha} X$ is not rimexcluded in $\alpha X$. Examples of this kind have to be found among rim-compact spaces with compactifications where the remainder is not zero-dimensionally embedded (Corollary 1 and Proposition 1). In particular all locally compact spaces with compactifications where the remainder is not zero-dimensional, give examples. In the following example, $X$ is not locally compact.

Example 2. Let $S, S^{0}, T$ and $X$ be as in Example 1. The space $S$ is a compactification $\alpha X$ of $X$. Clearly $X$ is rim-included in $\alpha X$, while $\rho_{\alpha} X$ contains $S-S^{0}$ and is thus not zero-dimensional.

Next consider the situation where $\rho_{\alpha} X$ is rim-excluded while $X$ is not rim-included in $\alpha X$. Examples of this kind have to be found among spaces with compactifications where the remainder is zero-dimensional but not zero-dimensionally embedded (Corollary 1). Such examples are not so easily constructed as the foregoing. We shall only indicate where to find relevant material.

Example 3. In [8] Yu. M. Smirnov described a space $X$ where (in our terminology) $\rho_{\beta} X$ is rim-excluded but not zero-dimensionally embedded in $\beta X$ (the Stone-Čech-compactification). Details of a similar construction is given in [5, p. 118].

We note that $X$ is not rim-compact. This is a consequence of the fact that for a rim-compact space, the maximal compactification with zero-dimensional remainder (which in this example evidently is $\beta X$ ) has zero-dimensionally embedded remainder (see, for example, [6, p 658]). 
Finally consider the situation where $X$ is not rim-included and $\rho_{a} X$ is not rim-excluded in $\alpha X$. Examples have to be found among spaces which are not locally compact, with compactifications where the remainder is not zero-dimensionally embedded. In the following example the space is rim-compact.

Example 4. Let $X$ be the spaces $S^{0} \cap T$, where $S^{0}$ and $T$ are as in Example 1, and let $\alpha X$ be the compactification $S$. Here $X$ is obviously not rim-included in $\alpha X$. Furthermore $\rho_{\alpha} X$ contains the connected set $S-S^{0}$, and is therefore not rim-excluded in $\alpha X$.

We note that the space $X$ is zero-dimensional, and thus in particular rimcompact.

\section{Preliminary results}

For further reference we discuss some simple relationships between sets in $X$ and in $\alpha X$.

Lemma 4. Let $C \in \mathcal{C}_{\alpha}$. Then $\operatorname{cl}_{\alpha}(C \cap X)=C$.

Proof. Obviously $\mathrm{cl}_{\alpha}(C \cap X) \subset C$. Let $x_{0} \in C \cap \rho_{\alpha} X$, and let $V$ be an arbitrary neighbourhood of $x_{0}$ in $\alpha X$. Since $C \in \mathcal{C}_{\alpha}, C$ is a neighbourhood of $x_{0}$ in $\alpha X$. Then $V \cap C \neq \varnothing$, hence $V \cap C \cap X \neq \varnothing$. This means that $x_{0} \in$ $\mathrm{cl}_{\alpha}(C \cap X)$, and consequently $C \cap \rho_{\alpha} X \subset \mathrm{cl}_{\alpha}(C \cap X)$. It follows that $C=(C$ $\cap X) \cup\left(C \cap \rho_{\alpha} X\right) \subset \mathrm{cl}_{\alpha}(C \cap X)$, which completes the proof.

Lemma 5. Let $B$ be a closed set in $X$, and let $C \in \mathcal{C}_{\alpha}$. Then $\operatorname{cl}_{\alpha}(B \cap C)=$ $\left(\mathrm{cl}_{\alpha} B\right) \cap C$.

Proof. Clearly $\operatorname{cl}_{\alpha}(B \cap C) \subset\left(\mathrm{cl}_{\alpha} B\right) \cap C$. Let $x_{0} \in\left(\mathrm{cl}_{\alpha} B\right) \cap C \cap \rho_{\alpha} X$. Since $C$ is a neighbourhood of $x_{0}$ and $x_{0} \in \mathrm{cl}_{\alpha} B$, we have $V \cap(B \cap C)=(V$ $\cap C) \cap B \neq \varnothing$ for every neighbourhood $V$ of $x_{0}$. Thus $\left(\operatorname{cl}_{\alpha} B\right) \cap C \cap \rho_{\alpha} X \subset$ $\mathrm{cl}_{\alpha}(B \cap C)$, and consequently

$$
\begin{aligned}
\left(\mathrm{cl}_{\alpha} B\right) \cap C & =\left(\mathrm{cl}_{\alpha} B \cap X \cap C\right) \cup\left(\mathrm{cl}_{\alpha} B \cap \rho_{\alpha} X \cap C\right) \\
& =(B \cap C) \cup\left(\mathrm{cl}_{\alpha} B \cap \rho_{\alpha} X \cap C\right) \subset \mathrm{cl}_{\alpha}(B \cap C) .
\end{aligned}
$$

This completes the proof.

We recall that a closed set $A$ is said to be regular closed if cl int $A=A$, and an open set $B$ is said to be regular open if int $\mathrm{cl} B=B$. 
Lemma 6. Let $A$ be a regular closed subset of $X$. Then $\mathrm{cl}_{\alpha} A$ is a regular (closed) subset of $\alpha X$.

Proof. Let $x_{0} \notin$ int $_{\alpha} \mathrm{cl}_{\alpha} A$, and let $V$ be an arbitrary neighbourhood of $x_{0}$. Then $V \cap\left(\alpha X-\operatorname{cl}_{\alpha} A\right) \neq \varnothing$, and hence $(X \cap V) \cap(\alpha X-A)=(X \cap V) \cap$ $\left(\alpha X-\mathrm{cl}_{\alpha} A\right)=X \cap\left[V \cap\left(\alpha X-\operatorname{cl}_{\alpha} A\right)\right] \neq \varnothing$. This means that $x_{0} \notin$ int $A$, and consequently int $A \subset$ int ${ }_{\alpha} \mathrm{cl}_{\alpha} A$. Taking into account the assumption cl int $A=A$, we obtain the inclusion $A=\operatorname{cl}$ int $A \subset \mathrm{cl}_{\alpha}$ int $A \subset \mathrm{cl}_{\alpha}$ int ${ }_{\alpha} \mathrm{cl}_{\alpha} A$. From this follows the desired inclusion $\mathrm{cl}_{\alpha} A \subset \mathrm{cl}_{\alpha}$ int $_{\alpha} \mathrm{cl}_{\alpha} A$.

We recall that a ring of sets is a family which is closed under finite unions and finite intersections. We note that $e_{\alpha}$ is a ring on $\alpha X$.

Let $\mathscr{F}$ be an arbitrary family of sets. Following [1] we denote by $\vee \mathcal{F}$ the family of all finite unions of sets in $\mathscr{F}$ and by $\wedge \mathscr{F}$ the family of all finite intersections of sets in $\mathscr{F}$. Then $\vee \wedge \mathscr{F}=\wedge \vee \mathscr{F}$ is the ring generated by $\mathscr{F}$.

We write $\mathrm{cl}_{\alpha} \mathscr{F}$ for the family $\left\{\mathrm{cl}_{\alpha} F: F \in \mathscr{F}\right\}$, where $\mathscr{F}$ is an arbitrary family of subsets of $X$.

LEMMA 7. Let $\mathcal{G}$ be a ring of closed subsets of $X$ contained in $\mathcal{C}_{\alpha} \cap X$. Then $\mathrm{cl}_{\alpha} \mathfrak{G}$ is a ring of closed subsets of $\alpha X$ contained in $\mathcal{C}_{\alpha}$.

Proof. By Lemma 4 we have $\mathrm{cl}_{\alpha} \mathcal{G} \subset \mathcal{C}_{\alpha}$. Clearly $\mathrm{cl}_{\alpha} \mathcal{G}$ is closed under finite unions. Let $G_{1}, G_{2} \in \mathcal{G}, G_{1}=H_{1} \cap X, G_{2}=H_{2} \cap X$, where $H_{1}, H_{2} \in \mathcal{C}_{\alpha}$. Let $x_{0} \in H_{1} \cap H_{2} \cap \rho_{\alpha} X$. As members of $\bigodot_{\alpha}$ the sets $H_{1}$ and $H_{2}$ and hence also $H_{1} \cap H_{2}$ are neighbourhoods of $x_{0}$. Let $V$ be an arbitrary neighbourhood of $x_{0}$. Then $V \cap\left(G_{1} \cap G_{2}\right)=\left(V \cap H_{1} \cap H_{2}\right) \cap X \neq \varnothing$, and consequently $x_{0} \in$ $\mathrm{cl}_{\alpha}\left(G_{1} \cap G_{2}\right)$. We conclude that $H_{1} \cap H_{2}=\left(H_{1} \cap H_{2} \cap X\right) \cup\left(H_{1} \cap H_{2} \cap\right.$ $\left.\rho_{\alpha} X\right) \subset \operatorname{cl}_{\alpha}\left(G_{1} \cap G_{2}\right)$, and hence $\mathrm{cl}_{\alpha} G_{1} \cap \mathrm{cl}_{\alpha} G_{2}=H_{1} \cap H_{2}=\operatorname{cl}_{\alpha}\left(G_{1} \cap G_{2}\right)$. It follows that $\operatorname{cl}_{\alpha} \mathcal{G}$ is closed under finite intersections, and so is a ring. This completes the proof.

LEMMA 8. Let $X$ be rim-included in $\alpha X$, and let $\mathcal{G}$ be a ring contained in $\mathcal{C}_{\alpha} \cap X$ which is a base for the closed sets on $X$. Let $K$ be a compact subset of $X$, and let $B$ be a closed subset of $\alpha X$ such that $K \cap B=\varnothing$. Then there exists an $F \in \mathrm{cl}_{\alpha} \mathcal{G}$ such that $B \subset F$ and $F \cap K=\varnothing$.

Proof. Let $x \in K$. By Lemma 2 there is a $G_{x} \in \mathcal{C}_{\alpha}$ such that $x \notin G_{x}$, $B \subset G_{x}$. Since $\mathcal{G}$ is a base for the closed sets on $X$, there is a $H_{x} \in \mathcal{C}_{\alpha}$ such that $H_{x} \cap X \in \mathcal{G}, x \notin H_{x}, H_{x} \cap X \supset G_{x} \cap X$. By Lemma 4, $\operatorname{cl}_{\alpha}\left(H_{x} \cap X\right)=H_{x}$, $\mathrm{cl}_{\alpha}\left(G_{x} \cap X\right)=G_{x}$, and hence $H_{x} \supset G_{x}$. Write $V_{x}=\alpha X-H_{x}$. The family 
$\left\{V_{x}: x \in K\right\}$ is an open covering of $K$. Let $\left\{V_{x_{1}}, \ldots, V_{x_{n}}\right\}$ be a finite sub-covering, and write $F=\alpha X-\bigcup_{i=1}^{n} V_{x_{i}}=\cap_{i=1}^{n} X_{x_{i}}$. Clearly $B \subset F$, and $F \cap K=$ $\varnothing$. Furthermore $H_{x} \in \mathrm{cl}_{\alpha} \mathcal{G}$, and hence by Lemma 7 also $F \in \mathrm{cl}_{\alpha} \mathcal{G}$. This completes the proof.

An s-ring $\mathscr{F}$ on $X$ is a ring of regular closed sets which is separating in the sense that if $x \notin A, A$ closed, then there are sets $F_{1}, F_{2} \in \mathcal{F}$ such that $x \in F_{1}$, $A \subset F_{2}, F_{1} \cap F_{2}=\varnothing$ (see [1, p. 126], [11, p. 297]). Since an $s$-ring evidently is a base for the closed sets, it follows that if an $s$-ring is contained in $\mathcal{C}_{\alpha} \cap X$, then $X$ is rim-included in $\alpha X$. A compact space which possesses an $s$-ring is called regular Wallman, and is a Wallman compactification of every dense subspace (see [11, p. 300]).

Let $\mathcal{F}$ be an $s$-ring on $S$. We define the family $\mathscr{F}_{\alpha}$ by

$$
\mathscr{F}_{\alpha}=\left\{F \cap C: F \in \mathscr{F}, C \in \mathcal{C}_{\alpha}, F \cap \partial_{\alpha} C=\varnothing\right\} .
$$

Clearly $\mathscr{F}_{\alpha}$ contains $\mathscr{F}$ (since $C=\alpha X \in \mathcal{C}_{\alpha}$ ), and is contained in $\mathcal{C}_{\alpha} \cap X$ if $\mathscr{F}$ is contained in $\mathcal{C}_{\alpha} \cap X$. We also note that the elements of $\mathscr{F}_{\alpha}$ are regular closed sets (since $F \cap C=F \cap$ int $_{\alpha} C$ ).

Proposition 2. Let $\mathscr{F}$ be an s-ring contained in $\mathcal{C}_{\alpha} \cap X$. Then $\vee \mathcal{F}_{\alpha}$ is an s-ring contained in $\mathcal{C}_{\alpha} \cap X$.

Proof. Since $\mathscr{F}_{\alpha}$ consists of regular closed sets, the same is true for $\bigvee \mathscr{F}_{\alpha}$. It is easily verified that $\mathscr{F}_{\alpha}$ is closed under finite intersections, and consequently $\vee \mathscr{F}_{\alpha}=\vee \wedge \mathscr{F}_{\alpha}$ is a ring. The ring $\vee \mathscr{F}_{\alpha}$ is separating since it contains the separating ring $\mathscr{F}$. Furthermore $\mathscr{F}_{\alpha} \subset \mathcal{C}_{\alpha} \cap X$, hence also $\vee \mathscr{F}_{\alpha} \subset \mathcal{C}_{\alpha} \cap X$. This completes the proof.

\section{Main results}

We now give conditions under which the existence of an $s$-ring on $X$ implies the existence of an $s$-ring on $\alpha X$.

THeOrem 1. Let $\rho_{\alpha} X$ be rim-excluded in $\alpha X$, and let $\mathscr{F}$ be an s-ring on $X$ contained in $\mathcal{C}_{\alpha} \cap X$. Then $\mathrm{cl}_{\alpha}\left(\vee \mathcal{F}_{\alpha}\right)$ is an s-ring on $\alpha X$.

Proof. From Lemma 6, Lemma 7 and Proposition 2 we conclude that $\mathrm{cl}_{\alpha}\left(\vee \mathscr{F}_{\alpha}\right)$ is a ring of regular closed sets on $\alpha X$. It remains to show that $\operatorname{cl}_{\alpha}\left(\vee \mathscr{F}_{\alpha}\right)$ is separating. 
We recall that since $\bigodot_{\alpha} \cap X$ contains an $s$-ring, $X$ is rim-included in $\alpha X$. As $\rho_{\alpha} X$ is assumed to be rim-excluded in $\alpha X, e_{\alpha}$ is a base for the closed sets on $\alpha X$ (Corollary 1). Let $x_{0} \in \alpha X, A$ closed in $\alpha X, x_{0} \notin A$. Let $V$ be a set in $\theta_{\alpha}$ such that $x_{0} \in V, A \cap \mathrm{cl}_{\alpha} V=\varnothing$. By Lemma 8 there is an $F \in \mathscr{F}$ such that $\left(\left\{x_{0}\right\} \cup A\right) \subset G, G \cap \partial_{\alpha} V=\varnothing$, where $G=\operatorname{cl}_{\alpha} F$. We observe that $\mathrm{cl}_{\alpha} V \in \mathcal{C}_{\alpha}$, $\operatorname{cl}_{\alpha}\left(\alpha X-\operatorname{cl}_{\alpha} V\right) \in \mathcal{C}_{\alpha}, \partial_{\alpha} \operatorname{cl}_{\alpha} V=\partial_{\alpha} \operatorname{cl}_{\alpha}\left(\alpha X-\operatorname{cl}_{\alpha} V\right)=\partial_{\alpha} V, F \cap \partial_{\alpha} V=\varnothing$. It follows that the sets $F_{1}=F \cap \mathrm{cl}_{\alpha} V$ and $F_{2}=F \cap \mathrm{cl}_{\alpha}\left(\alpha X-\mathrm{cl}_{\alpha} V\right)$ belong to $\mathscr{F}_{\alpha}$. By using Lemma 5 we get

$$
x_{0} \in G \cap \mathrm{cl}_{\alpha} V=\mathrm{cl}_{\alpha} F \cap \mathrm{cl}_{\alpha} V=\mathrm{cl}_{\alpha}\left(F \cap \mathrm{cl}_{\alpha} V\right)=\mathrm{cl}_{\alpha} F_{1},
$$

and

$$
A \subset G \cap \mathrm{cl}_{\alpha}\left(\alpha X-\mathrm{cl}_{\alpha} V\right)=\mathrm{cl}_{\alpha} F \cap \mathrm{cl}_{\alpha}\left(\alpha X-\mathrm{cl}_{\alpha} V\right)=\operatorname{cl}_{\alpha} F_{2} .
$$

Furthermore $\left(\mathrm{cl}_{\alpha} F_{1} \cap \mathrm{cl}_{\alpha} F_{2}\right) \cap \rho_{\alpha} X=\varnothing$ and $F_{1} \cap F_{2}=\varnothing$ (since $F \cap \partial_{\alpha} V=$ $\varnothing)$. Thus $\mathrm{cl}_{\alpha} F_{1} \cap \mathrm{cl}_{\alpha} F_{2}=\varnothing$. Finally we evidently have $\operatorname{cl}_{\alpha} F_{1}, \operatorname{cl}_{\alpha} F_{2} \in \operatorname{cl}_{\alpha} \mathscr{F}$ $\subset \mathrm{cl}_{\alpha}\left(\vee \mathscr{F}_{\alpha}\right)$. This completes the proof.

We shall discuss some implications of Theorem 1 for the problem of deciding whether a compactification is regular Wallman.

For the sake of completeness we give a proof of the following easy result.

Proposition 3. If $\gamma X \leqslant \alpha X$, then $\mathcal{\theta}_{\gamma} \cap X \subset \mathcal{O}_{\alpha} \cap X$. Hence if $X$ is rim-included in a compactification $\gamma X$, then $X$ is also rim-included in every larger compactification $\alpha X$.

PRoOf. Let $q$ be the quotient map of $\alpha X$ onto $\gamma X$. For every $O \in \mathcal{\theta}_{\gamma}$, $U=q^{-1}(0)$ is open, and $U \cap X=O \cap X$. Let $y \in \partial_{\alpha} U$. Then $q(y) \in \partial_{\gamma} O \subset$ $X$, hence $y=q(y) \in X$. Thus $\partial_{\alpha} U \subset X$, or $O \cap X=U \cap X \in \mathcal{\theta}_{\alpha} \cap X$. Consequently $\mathcal{\theta}_{\gamma} \cap X \subset \mathcal{\theta}_{\alpha} \cap X$, from which the result follows.

TheOREM 2. Let $\mathcal{F}$ be an s-ring in $\mathcal{C}_{\gamma} \cap X$ for some compactification $\gamma X$. Then for every larger compactification $\alpha X$ where $\rho_{\alpha} X$ is rim-excluded, $\operatorname{cl}_{\alpha}\left(\vee \mathscr{F}_{\alpha}\right)$ is an s-ring on $\alpha X$.

Proof. Immediate by Proposition 3 and Theorem 1.

COROLlary 2. Assume that $X$ has a compactification $\gamma X$ where $\mathcal{C}_{\gamma}$ contains an $s$-ring. Then every larger compactification $\alpha X$ for which $\rho_{\alpha} X$ is rim-excluded, is regular Wallman. 
Proof. Let $\mathcal{G}$ be an $s$-ring contained in $\mathcal{C}_{\gamma}$. It is easily verified that $\mathcal{G} \cap X$ is an $s$-ring on $X$. The result now follows from Theorem 2 .

Remark 2. Theorem 2 (and Corollary 2) can only give a positive answer to the question whether a compactification $\alpha X$ is regular Wallman in cases where the space $X$ is rim-compact and the remainder $\rho_{\alpha} X$ is zero-dimensionally embedded. For these conditions are clearly contained in the assumptions of the theorem.

We shall say that the space $X$ is totally rim-included in the compactification $\alpha X$ if every point $x \in X$ has a neighbourhood $V_{x}$ in $X$ such that every regular open subset of $V_{x}$ belongs to $\mathcal{\theta}_{\alpha} \cap X$. Every dense subspace of an extremally disconnected compact space $K$ is totally rim-included in $K$. (In an extremally disconnected space the closure of every open set is open.) A locally compact space is obviously totally rim-included in every compactification.

We note that according to Proposition 3, if $X$ is totally rim-included in some compactification, then $X$ is also totally rim-included in every larger compactification.

LEMMA 9. Let $X$ have a compactification $\gamma X$ such that $X$ is totally rim-included in $\gamma X$. If $X$ possesses an s-ring, then $X$ possesses an s-ring contained in $\mathcal{C}_{\gamma} \cap X$.

Proof. Let $\mathcal{G}$ be an $s$-ring on $X$, and define $\mathscr{F}=\mathcal{G} \cap\left(\mathcal{C}_{\gamma} \cap X\right)$. Evidently $\mathscr{F}$ is a ring contained in $\mathcal{C}_{\gamma} \cap X$ consisting of regular closed sets. It remains to show that $\mathscr{F}$ is separating.

Let $x_{0} \in X, x_{0} \notin A, A$ closed in $X$. There is a set $V$ in $\theta_{\gamma}$ such that $x_{0} \in V$ and $\operatorname{cl}_{\gamma} V \cap A=\varnothing$, and such that every regular open subset of $V \cap X$ belongs to $\mathcal{O}_{\gamma} \cap X$. The set $B=\gamma X-V$ belongs to $\mathcal{C}_{\gamma}$. Since $\mathcal{G}$ is an $s$-ring, there are sets $F_{1}$ and $F_{2}$ in $\mathcal{G}$ such that $x_{0} \in F_{1}, B \cap X \subset F_{2}, F_{1} \cap F_{2}=\varnothing$. Now $X-F_{2}$ is a regular open subset of $V \cap X$, hence $X-F_{2} \in \mathcal{O}_{\gamma} \cap X$, and so $F_{2} \in \mathcal{C}_{\gamma} \cap$ $X$. Similarly int $F_{1}$ is a regular open subset of $V \cap X$, hence int $F_{1} \in \mathcal{O}_{\gamma} \cap X$. Let int $F_{1}=O \cap X, O \in \mathcal{O}_{\gamma}$. Then $\operatorname{cl}_{\gamma} O \in \mathcal{C}_{\gamma}$, and $\left(\mathrm{cl}_{\gamma} O\right) \cap X=\operatorname{cl}(O \cap X)$ (since $X$ is dense in $\gamma X$ ). Thus $F_{1}=\operatorname{cl}$ int $F_{1}=\left(\operatorname{cl}_{\gamma} O\right) \cap X \in \mathcal{C}_{\gamma} \cap X$. It follows that $F_{1}, F_{2} \in \mathcal{G} \cap\left(\mathcal{C}_{\gamma} \cap X\right)=\mathscr{F}$, which shows that $\mathscr{F}$ is separating.

This completes the proof.

From Lemma 9 and Theorem 2 we immediately obtain the following corollary.

Corollary 3. Assume that $X$ has a compactification $\gamma X$ such that $X$ is totally rim-included in $\gamma X$. If $X$ possesses an s-ring, then every compactification $\alpha X$ where $\alpha X$ is larger than $\gamma X$ and $\rho_{\alpha} X$ is rim-excluded in $\alpha X$, is regular Wallman. 
We shall here briefly discuss the situation for locally compact spaces. Let $\omega X$ denote the one-point compactification. Every compactification $\alpha X$ is larger than $\omega X$. We have already noted that $X$ is totally rim-included in $\omega X$, in fact, in every compactification $\alpha X$. If $\rho_{\alpha} X$ is zero-dimensional, then $\rho_{\alpha} X$ is also rim-excluded in $\alpha X$. So Corollary 3 contains the following result of Baayen and van Mill ([1, Theorems 2 and 3]), the proof of which gave rise to some of the main ideas of this paper:

THEOREM 3. Let $X$ be a locally compact space which possesses an s-ring. Then every compactification $\alpha X$ where $\rho_{\alpha} X$ is zero-dimensional, is regular Wallman.

\section{Some applications}

It may be of interest to discuss some intrinsic conditions on the space $X$ which insure that $X$ is totally rim-included in some compactification (or in some class of compactifications).

We recall that the Freudenthal compactification $\phi X$ of a rim-compact space may be obtained as the Wallman compactification corresponding to the normal base $\Re$ consisting of all finite intersections of regular closed sets with compact boundaries (see, for example, [2, p. 108-112]). Every compactification with zero-dimensionally embedded remainder may be obtained as the Wallman compactification corresponding to a normal base $\mathscr{Q}$ contained in $\Re$ and having the property: $A \in \mathbb{Q}$ implies $X-$ int $A \in \mathbb{Q}$. Every normal base of this kind contained in $\Re$ (on a rim-compact space) gives rise to a Wallman compactification $\alpha X$ with zero-dimensionally embedded remainder. More precisely the basic sets $A^{*}$ on $\alpha X$ corresponding to sets $A$ in $\mathcal{Q}$ have their boundaries in $X$, which means that $\mathscr{Q} \subset \mathcal{C}_{\alpha} \cap X$, or $\tilde{\mathbb{Q}}=\{X-A: A \in \mathbb{Q}\} \subset \mathcal{\theta}_{\alpha} \cap X$. (See [3, p. 6566].) Now in addition assume that $\mathscr{Q}$ contains a base $\mathscr{Q}$ such that every regular closed set which contains a set in $\mathscr{B}$ belongs to $\mathscr{B}$. Then the family $\tilde{\mathscr{B}}=\{X-$ $B: B \in \mathscr{B}\}$ is a base for the open sets, and every regular open subset of a set in $\tilde{\mathscr{B}}$ belongs to $\tilde{\mathscr{B}}$. It follows from the remarks above that $\tilde{\mathscr{B}} \subset \mathcal{O}_{\alpha} \cap X$, which means that $X$ is totally rim-included in $\alpha X$. From Corollary 3 and the fact that $\rho_{\alpha} X$ is zero-dimensionally embedded in $\alpha X$, we may thus conclude:

Proposition 4. Let $\mathbb{Q}$ be a normal base on the rim-compact space $X$, contained in $\mathfrak{R}$. Assume that $\mathbb{Q}$ has the property: $A \in \mathbb{Q}$ implies $X-$ int $A \in \mathbb{Q}$. Further assume that $\mathbb{Q}$ contains a base $\mathscr{B}$ such that every regular closed set containing $a$ set in $\mathscr{B}$ belongs to $\mathscr{B}$. Then $\alpha X$ is regular Wallman if $X$ possesses an $s$-ring. 
We shall say that $X$ is totally rim-compact if for every $x \in X$ there is a neighbourhood $V_{x}$ such that the boundary of every regular open subset of $V_{x}$ is compact. Evidently every locally compact space and every extremally disconnected space is totally rim-compact.

If $X$ is totally rim-compact, then the normal base $R$ clearly satisfies the conditions of Proposition 4. Thus we may state the following corollary.

COROLlaRY 4. If a totally rim-compact space has an s-ring, then the Freudenthal compactification $\phi X$ is regular Wallman.

We shall now discuss certain classes of totally rim-compact spaces in some detail.

We shall first describe a (rather special) type of space which is totally rim-compact but neither locally compact nor extremally disconnected. Let $X$ be a space where all points are open, except for one point $x_{0}$. Assume that there is a set $A_{0} \subset X-\left\{x_{0}\right\}$ such that neither $\left\{x_{0}\right\} \cup A_{0}$ nor $X-A_{0}$ is open. Further assume that for every neighbourhood $V$ of $x_{0}$ there is a neighbourhood $W$ of $x_{0}$ such that $V-W$ is infinite. In this situation we easily verify that $X$ is totally rim-compact (every neighbourhood of $x_{0}$ has empty boundary), not locally compact (every neighbourhood $V$ of $x_{0}$ contains a closed subset $V-W$ which is not compact), and not extremally disconnected (the closure of the open set $A_{0}$ is $\left\{x_{0}\right\} \cup A_{0}$, which is not open).

A space which satisfies the conditions described is the Appert space (see $[9, \mathrm{p}$. 117-118]):

EXample 5. Let $X=N$ (the set of positive integers). For every $n \in N$ let $\nu(n, E)$ denote the number of integers in the set $E$ which is less than or equal to $n$. Every integer $2,3,4, \ldots$ is an open set, and the neighbourhoods of 1 are the sets $E$ containing 1 for which $\lim _{n \rightarrow \infty} \nu(n, E) / n=1$. This space is easily seen to have the properties required.

We shall next indicate a more general method for obtaining totally rim-compact spaces which are neither locally compact nor extremally disconnected.

Let $D$ be a subset of a topological space $X$. We define the $D$-discrete modification $X_{D}$ of $X$ as the topological space having the same points as $X$, with all points of $X-D$ having their original neighbourhoods and all points of $D$ being open. $X_{D}$ is easily seen to be a completely regular Hausdorff space when $X$ is. 
Proposition 5. Let $D$ be a subset of the space $X$ such that $X-D$ is a closed nowhere dense subset which is locally compact as a subspace. Then $X_{D}$ is totally rim-compact.

If in the subspace $D$ of $X$ the set of non-isolated points is dense, then $X_{D}$ is not locally compact.

If there is a point in $X-D$ which is a boundary point for an open set in $X$, then $X_{D}$ is not extremally disconnected.

PRoof. Let $\mathrm{cl}_{D}$ and $\partial_{D}$ denote closure and boundary in the space $X_{D}$.

Let $V$ be a neighbourhood of a point $x \in X-D$ such that cl $V \cap(X-D)$ is compact. Let $W$ be an arbitrary subset of $V$. Then clearly $\partial_{D} W \subset \operatorname{cl} V \cap(X$ $-D$ ), hence $\partial_{D} W$ is compact (in the topology induced from $X_{D}$, or from $X$ ). Thus $X_{D}$ is totally rim-compact.

Assume that the set of non-isolated points of the subspace $D$ of $X$ is dense in $D$. Let $x \in X-D$, and let $U$ be an arbitrary neighbourhood of $x$. Then $U \cap D \neq \varnothing$ since $X-D$ is nowhere dense in $X$. Let $y \in U$ be non-isolated in $D$, and let $V$ be a neighbourhood of $y$ such that $\operatorname{cl} V \subset D$. Clearly cl $V$ is infinite, hence not compact in $X_{D}$. Thus every neighbourhood of $x$ contains a closed non-compact subset. It follows that $X_{D}$ is not locally compact.

Assume that the point $x_{0} \in X-D$ is a boundary point of some open set $G$ in $X$. Then $x_{0}$ is also a boundary point of $G$ in $X_{D}$. It follows that $\mathrm{cl}_{D} G$ is not open, and so $X_{D}$ is not extremally disconnected.

This completes the proof.

EXAMPLE 6. Instances of the situation described above are easily obtained in the spaces $R^{n}$. Let for example: i) $X=R^{1}, X-D=$ the Cantor set $C$, or ii) $X=C \cup Q\left[Q\right.$ denotes the rationals], $X-D=C$, or iii) $X=R^{2}, X-D=$ the diagonal $\Delta=\left\{(x, y) \in R^{2}: x=y\right\}$, or: iv) $X=R^{2}, X-D=Z \times Z[Z$ denotes the integers], or: v) $X=Q \times Q, X-D=Z \times Z$, or vi) $X=(Q \times$ $Q) \cup \Delta, X-D=\Delta$, or vii) $x=R^{3}, X-D=R^{2} \times\{0\}$.

In these cases, all the conditions of Proposition 5 are easily seen to be satisfied.

We shall show that the Freudenthal compactification of some totally rimcompact spaces obtained by the procedure described above, is regular Wallman. Some of the spaces indicated in Example 6 are among those considered in the following proposition.

Proposition 6. Let $X$ be a locally compact metric space and let $D$ be any subset such that $X-D$ is nowhere dense and the distance between two arbitrary points of 
$X-D$ is at least 1. Then the $D$-modified space $X_{D}$ is totally rim-compact, and its Freudenthal compactification is regular Wallman.

Proof. It follows from Proposition 5 that $X_{D}$ is totally rim-compact.

Let $\mathscr{F}$ be the family consisting of all finite subsets of $D$, of the complements of all such sets, of all sets $V-F$, where $V$ is a closed disc in $X$ with center at some point of $X-D$ and radius less than $\frac{1}{2}$, and $F$ is a finite subset of $D$, and of the complements of all such sets. The family $\wedge \mathscr{F}$ and hence also the family $\vee \wedge \mathscr{F}$ is easily seen to consist of regular closed sets in $X_{D}$. (Actually the sets in $\vee \wedge \mathscr{F}$ are open-closed.) Furthermore $\mathscr{F}$ and hence $\vee \wedge \mathscr{F}$ is clearly separating. Thus $\vee \wedge \mathscr{F}$ is an $s$-ring on $X_{D}$. It follows from Corollary 4 that $\phi X_{D}$ is regular Wallman.

\section{References}

[1] P. C. Baayen and J. van Mill, 'Compactifications of locally compact spaces with zero-dimensional remainder', General Topology and Appl. 9 (1978), $125-129$.

[2] B. Banaschewski, 'On Wallman's method of compactification', Math. Nachr. 27 (1963), $105-114$.

[3] J. Flachsmeyer, 'Über Erweiterungen mit nulldimensional gelegenem Adjunkt', Contributions to Extension Theory of Topological Structures (Academic Press 1969, 63-72).

[4] H. Freudenthal, 'Kompaktifizierungen und Bikompaktifizierungen', Indag. Math. 13 (1951), $184-192$.

[5] J. R. Isbell, Uniform spaces (American Mathematical Society, Providence, Rhode Island, (1964)).

[6] J. R. McCartney, 'Maximum zero-dimensional compactifications', Proc. Cambridge Philos. Soc. 68 (1970), 653-661.

[7] K. Morita, 'On bicompactifications of semicompact spaces', Sci. Rep. Tokyo Bunrika Daigaku 4 (1952), 222-229.

[8] Yu. M. Smirnow, 'A completely regular non-semibicompact space with a zero-dimensional Cech complement', Dokl. Akad. Nouk. SSSR 120 (1958), 1204-1206 (Russian).

[9] L. A. Steen and J. A. Seebach, Counterexamples in topology (2nd ed., Springer-Verlag, New York-Heidelberg-Berlin, (1978)).

[10] A. K. Steiner and E. F. Steiner, 'Wallman and Z-compactifications', Duke Math. J. 35 (1968), 269-275.

[11] E. F. Steiner, 'Wallman spaces and compactifications', Fund. Math. 61 (1968), 295-304.

\section{Department of Mathematics}

University of Trondheim-Norwegian

Institute of Technology

N-7034 Trondheim

Norway 